\title{
Antiplatelet Therapy in Patients with Aneurysmal SAH: Impact on Delayed Cerebral Ischemia and Clinical Outcome. A Meta-Analysis
}

(D) F. Cagnazzo, (DI. Derraz, (DP.-H. Lefevre, (D). Gascou, (D). Dargazanli, (D). Riquelme, (DP. Perrini, (D) D. di Carlo, (D)A. Bonafe, and (1) V. Costalat

\begin{abstract}
BACKGROUND AND PURPOSE: Delayed cerebral ischemia strongly impacts clinical outcome after aneurysmal SAH. The effect of antiplatelet therapy on delayed cerebral ischemia has been described with heterogeneous results. Our aim was to analyze the efficacy of antiplatelet therapy on delayed cerebral ischemia and clinical outcome in patients with SAH.
\end{abstract}

DATA SOURCES: A systematic search of 3 databases was performed for studies published from 1990 to 2019.

STUDY SELECTION: According to the Preferred Reporting Items for Systematic Reviews and Meta-Analyses guidelines, we included studies comparing the rates of delayed cerebral ischemia and clinical outcomes among patients with SAH with and without antiplatelet therapy.

DATA ANALYSIS: Random-effects meta-analysis was used to pool the following: delayed cerebral ischemia, mortality, and good outcome rates.

DATA SYNTHESIS: Including 7 studies, 1060 and 1762 patients with SAH were endovascularly or surgically treated with (cases) and without (controls) antiplatelet therapy, respectively. Overall, antiplatelet therapy did not significantly decrease delayed cerebral ischemia rates compared with the control group (219/1060 versus $485 / 1762, \mathrm{OR}=0.781 ; 95 \% \mathrm{Cl}, 0.46-1.31 ; P=.33)$. Among patients treated endovascularly, there was a trend toward lower delayed cerebral ischemia rates after antiplatelet therapy $(157 / 778$ versus $413 / 1410,0 R=0.552 ; 95 \% \mathrm{Cl}$, 0.273-1.115; $P=.06$ ). Long-term ( $>2$ weeks) antiplatelet therapy tended to be associated with a lower incidence of delayed cerebral ischemia (63/438 versus $96 / 353, \mathrm{OR}=0.379 ; 95 \% \mathrm{Cl}, 0.12-1.2 ; P=.06)$. The good-outcome rate was significantly higher ( $803 / 1144$ versus $1175 / 1775, \mathrm{OR}=1.368 ; 95 \% \mathrm{Cl}, 1.117-1.676 ; P=.002)$ and the mortality rate was significantly lower $(79 / 672$ versus $97 / 571, \mathrm{OR}=0.656 ; 95 \% \mathrm{Cl}$, $0.47-0.91 ; P=.01$ ) among the antiplatelet therapy group.

LIMITATIONS: Heterogeneity was high for most outcomes.

CONCLUSIONS: Overall, the incidence of delayed cerebral ischemia seems not to be significantly reduced among the antiplatelet therapy group. However, delayed cerebral ischemia tended to be lower among subjects with both long-term antiplatelet therapy and endovascular treatment and antiplatelet administration. Poor outcome and mortality rates were significantly reduced among the antiplatelet therapy group.

ABBREVIATIONS: $\mathrm{ASA}=$ acetylsalicylic acid; $\mathrm{AT}=$ antiplatelet therapy; $\mathrm{CV}=$ cerebral vasospasm; $\mathrm{DCl}=$ delayed cerebral ischemia

A mong patients with aneurysmal subarachnoid hemorrhage, delayed cerebral ischemia (DCI) represents a devastating complication that occurs in approximately $30 \%$ of subjects who survive the initial bleeding. ${ }^{1}$ In general, DCI is defined as the development of new neurologic deficits, occurring most often

Received March 3, 2019; accepted after revision April 25.

From the Neuroradiology Department (F.C., I.D., P.-H.L., G.G., C.D., C.R., A.B., V.C.), University Hospital Güi-de-Chauliac, Centre Hospitalier Universitaire de Montpellier, Montpellier, France; and Department of Neurosurgery (P.P., D.d.C.), University of Pisa, Pisa, Italy.

Please address correspondence to Federico Cagnazzo, MD, Neuroradiology Department, CHU Gui De Chauliac, 80 Ave Augustin Fliche, 34000 Montpellier, France; e-mail: f.cagnazzo86@gmail.com between 4 and 10 days after SAH, confirmed by new cerebral hypodense lesions on CT with no evidence of hydrocephalus or rebleed. $^{2-5}$ The pathogenesis of DCI is still not completely clarified, and several mechanisms have been proposed, such as inflammatory activation, vasospasm, cortical spreading depolarization, and microthrombosis. ${ }^{1}$ Along with the above-mentioned mechanisms, an increased platelet activity after SAH might also be involved in the development of DCI. ${ }^{6,7}$ Platelet aggregability and

Indicates article with supplemental on-line tables.

Indicates article with supplemental on-line photos.

http://dx.doi.org/10.3174/ajnr.A6086 
the associated release of thromboxane $\mathrm{B} 2$ are increased in patients with SAH, especially in subjects with DCI. ${ }^{3}$ Accordingly, several studies indicated a possible preventive effect of antiplatelet therapy (AT), especially with aspirin, on the development of DCI in patients with SAH. However, the results are quite heterogeneous, and the efficacy of the platelet inhibitors for the prevention of DCI is still debated. Recently, 2 series of ruptured intracranial aneurysms treated by endovascular means showed that antiplatelet agents effectively reduced the risk of vasospasm and DCI. ${ }^{2,8}$ Our meta-analysis aimed to investigate the effect of antiplatelet drug administration on the prevention of DCI among patients with aneurysmal SAH. In addition, including recent series of endovascular treatments, we explored the effect of antiplatelet inhibitors in relation to the type of treatment.

\section{MATERIALS AND METHODS Literature Search}

A comprehensive literature search of PubMed, Ovid EMBASE, and Scopus was conducted for studies published from January 1990 to January 2019. The Preferred Reporting Items for Systematic Reviews and Meta-Analyses (PRISMA) guidelines ${ }^{9}$ were followed. The key words and the detailed search strategy are reported in On-line Table 1, and the studies included in our review are reported in On-line Table 2. The main inclusion criteria were the following: 1) studies reporting patients with aneurysmal SAH who were treated with (cases) and without (controls) AT during the acute phase, 2) studies reporting clinical outcomes (incidence of DCI, cerebral vasospasm [CV], and neurologic outcome) of patients with aneurysmal SAH undergoing treatment with AT. In addition, the selection was restricted to studies reporting treatment with acetylsalicylic acid (ASA) (or ASA plus clopidogrel) given during the acute phase of SAH for the period of risk for CV and DCI (AT was continued for at least 2-3 weeks after SAH). Exclusion criteria were the following: 1) case reports, 2) review articles, 3) studies published in languages other than English, 4) in vitro/animal studies, and 5) studies reporting patients treated with other antiplatelet drugs different from ASA or clopidogrel, and 6) studies reporting patients for whom the AT was not continued during the period of risk for DCI and CV. In cases of overlapping patient populations, only the series with the largest number of patients or the most detailed data was included. Two independent readers screened articles in their entirety to determine eligibility for inclusion. A third author solved discrepancies.

\section{Data Collection}

We extracted the following: 1) incidence of DCI, 2) incidence of $\mathrm{CV}, 3$ ) treatment-related complications, and 4) clinical outcome. The reported results were compared between patients treated (cases) and not treated (controls) with AT.

DCI was defined as the occurrence of new clinical features suggestive of DCI (gradually developed focal deficits, a decreased level of consciousness, or both) confirmed by new cerebral hypodense lesions on CT with no evidence of hydrocephalus or rebleed. $^{2-5}$ Good outcome was defined as a modified Rankin Scale score of $0-2$ or a Glasgow Outcome Score of 4-5.

\section{Outcomes}

The primary objective of this study was to compare the incidence of DCI and good neurologic outcome between the AT and the control groups. The secondary objectives were to define the following: 1) the incidence of CV among the AT and the control groups, 2) the influence of AT on the occurrence of DCI based on the type of aneurysm treatment (endovascular versus surgical), and 3 ) the influence of the duration (short-term, $<2$ weeks versus long-term, $>2$ weeks) of the AT on the DCI rate.

\section{Quality Scoring}

The Newcastle-Ottawa Scale ${ }^{10}$ was used for the quality assessment of the included studies (details in On-line Table 3). The quality assessment was performed by 2 authors independently, and a third author solved discrepancies.

\section{Statistical Analysis}

We estimated, from each cohort, the cumulative prevalence (percentage) and 95\% confidence interval for each outcome. Heterogeneity of the data was assessed $\mathrm{I}^{2}$, and subsequently, the DerSimonian and Laird random-effects model was applied. The graphic representation was performed by a forest plot. To evaluate the heterogeneity and bias, we analyzed the meta-regression and funnel plot followed by the Egger linear regression test, respectively. To verify the consistency of outcome meta-analysis results, the influence of each individual study on the summary effect estimate was assessed by the sensitivity analysis ("leave-oneout" approach) and the subgroups analysis. Differences were considered significant at $P<.05$. Meta-analysis was performed with ProMeta-2 (Internovi, Cesena, Italy) and OpenMeta[Analyst] (http://www.cebm.brown.edu/openmeta/).

\section{RESULTS}

\section{Literature Review}

Studies included in our meta-analysis are summarized in On-line Table 2. The search flow diagram is shown in On-line Fig 1.

A total of 7 studies and 2822 patients with aneurysmal SAH were included in our review. Overall, 1060 patients were treated with AT (cases), whereas 1762 patients did not receive antiplatelet agents (controls). All the included studies compared patients with $\mathrm{SAH}$ with and without AT during the acute phase.

\section{Quality of Studies}

Five studies presented a prospective design ${ }^{3-5,11,12}$ : Two studies were randomized controlled trials, ${ }^{3,5} 1$ study was prospective, ${ }^{4}$ and 2 series were post hoc analyses from prospective randomized trials. ${ }^{11,12}$ Two studies had a retrospective design. ${ }^{2,8}$ Overall, all the reported articles were rated as "high-quality" studies based on the Newcastle-Ottawa Scale criteria.

\section{Patient Population Characteristics}

Detailed characteristics of the patient population are reported in On-line Tables 2 and 4 . The mean age of patients was comparable between the 2 groups. The proportion of male patients was higher among the control group (49.9\%; 95\% CI, 47\%-51\% versus $43.7 \%$; 95\% CI, 41\%-47\%; $P=.006$ ). The proportion of highgrade SAH (Fisher 3-5) was slightly higher among the control 
Darkwah Oppong et al, 2018

Nagahama et al, 2018

van den Bergh et al (endovascular) 2009

van den Bergh et al (from ISAT study) 2009

Hop et al, 2000

Juvela, 1995

van den Bergh et al (MASH study) 2006

van den Bergh et al (surgical) 2009

Overall $\left(I^{2}=76.94 \%, P<.001\right)$

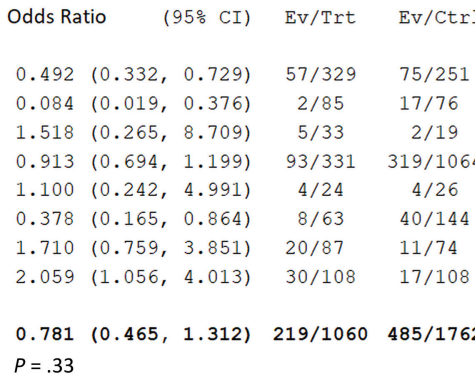

$P=.33$

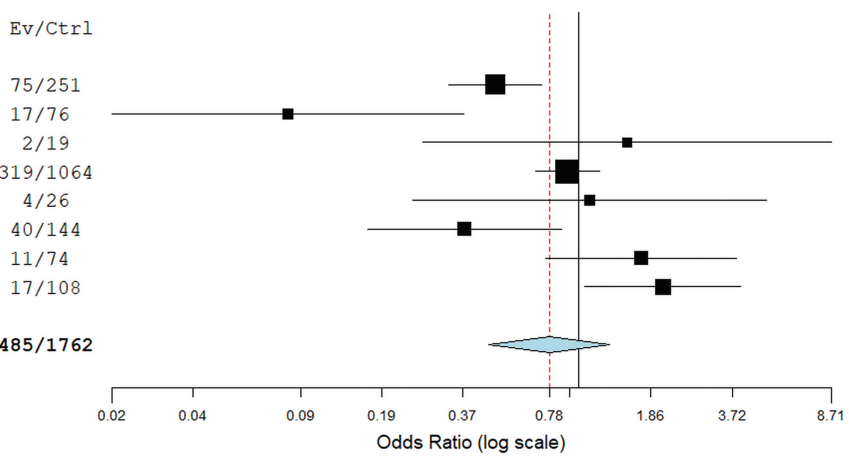

FIG 1. Forest plot with a random-effects model demonstrating the overall incidence (odds ratio meta-analysis) of DCI in the AT group compared with the control group. Ev indicates Events; Trt, Treated patients; Ctrl, Control.

Clinical outcomes after subarachnoid hemorrhage: comparison between the antiplatelet and the control groups

\begin{tabular}{|c|c|c|c|}
\hline Outcomes & Antiplatelet Group vs Control Group $(95 \% \mathrm{CI})\left(\mathrm{I}^{2}\right)$ & $\begin{array}{c}\text { No. of } \\
\text { Articles }\end{array}$ & $\begin{array}{c}P \\
\text { Value }\end{array}$ \\
\hline Overall rate of delayed cerebral ischemia & $219 / 1060$ vs $485 / 1762, \mathrm{OR}=0.781(95 \% \mathrm{Cl}, 0.46-1.31)\left(I^{2}=76.9 \%\right)$ & 7 & .33 \\
\hline Delayed cerebral ischemia endovascular group & $157 / 778$ vs $413 / 1410, \mathrm{OR}=0.552(95 \% \mathrm{Cl}, 0.273-1.115)\left(1^{2}=80.1 \%\right)$ & 4 & $.06^{\mathrm{a}}$ \\
\hline Delayed cerebral ischemia surgical group & $42 / 195$ vs $61 / 278, \mathrm{OR}=0.952(95 \% \mathrm{Cl}, 0.288-3.147)\left(1^{2}=79.5 \%\right)$ & 3 & .5 \\
\hline Delayed cerebral ischemia long-term $\mathrm{AT}^{\mathrm{b}}$ & $63 / 438$ vs $96 / 353, \mathrm{OR}=0.379(95 \% \mathrm{Cl}, 0.12-1.2)\left(1^{2}=68 \%\right)$ & 3 & $.06^{\mathrm{a}}$ \\
\hline Delayed cerebral ischemia short-term $\mathrm{AT}^{\mathrm{C}}$ & $148 / 559$ vs $349 / 1265, \mathrm{OR}=1.35(95 \% \mathrm{Cl}, 0.81-2.23)\left(1^{2}=80.1 \%\right)$ & 4 & .5 \\
\hline Cerebral vasospasm & $79 / 414$ vs $84 / 327, \mathrm{OR}=0.52(95 \% \mathrm{Cl}, 0.2-1.5)\left(1^{2}=83 \%\right)$ & 2 & .2 \\
\hline Good outcome & $803 / 1144$ vs $1175 / 1775$, OR $=1.368(95 \% \mathrm{Cl}, 1.117-1.676)\left(I^{2}=9.3 \%\right)$ & 8 & $.002^{\mathrm{a}}$ \\
\hline Mortality & $79 / 672$ vs $97 / 571, \mathrm{OR}=0.656(95 \% \mathrm{Cl}, 0.47-0.91)\left(1^{2}=0 \%\right)$ & 5 & $.01^{\mathrm{a}}$ \\
\hline Overall rate of complications & $40 / 438$ vs $28 / 353, \mathrm{OR}=1.01(95 \% \mathrm{Cl}, 0.34-3.01)\left(1^{2}=42 \%\right)$ & 3 & .9 \\
\hline Overall rate of hemorrhagic complications & $32 / 438$ vs $15 / 353, \mathrm{OR}=1.7(95 \% \mathrm{Cl}, 0.89-3.38)\left(1^{2}=1 \%\right)$ & 3 & .1 \\
\hline Overall rate of major hemorrhagic events & $8 / 438$ vs $8 / 353, \mathrm{OR}=0.857(95 \% \mathrm{Cl}, 0.32-2.28)\left(I^{2}=10\right)$ & 3 & .7 \\
\hline
\end{tabular}

a Significant.

${ }^{\mathrm{b}}$ Long-term AT $=$ AT for 3 or 4 weeks

' Short-term AT $=$ AT for $<2$ weeks.

group $(67.7 \%$; 95\% CI, 65.6\%-70\% versus $60.6 \%$; 95\% CI, $57 \%-$ $63.5 \% ; P=.001)$. There were no aneurysms treated with stentassisted coiling or flow diversion among the control group, whereas patients treated with coiling were slightly lower in the AT group $(63.3 \%$; 95\% CI, 60\%-66\%) compared with controls (74\%; 95\% CI, 72\%-76\%; $P=.001)$.

In all except 1 study, the AT consisted of ASA, $100 \mathrm{mg} /$ day, started after the aneurysm occlusion (On-line Table 2). Dual AT was used in the series of Nagahama et $\mathrm{al}^{8}$ (clopidogrel, $600 \mathrm{mg}$, + ASA, 325/day, after aneurysm embolization). In 1 study, ${ }^{11}$ the dose of ASA ranged between $75 \mathrm{mg}$ and $325 \mathrm{mg}$ /day.

The mean clinical follow-up was 5 months (median, 3.5 months; interquartile range, 2-6 months).

\section{Treatment-Related Outcomes among the AT and Control Groups}

Treatment-related outcomes were analyzed with the random-effects model because this model incorporates heterogeneity among studies. Overall, there was not a statistically significant reduction of the rate of DCI among the AT group compared with the control group $(219 / 1060$ versus $485 / 1762$, OR $=0.781 ; 95 \% \mathrm{CI}, 0.46-$ 1.31; $P=.33$ ) (Fig 1 and Table). The funnel-plot, followed by the Egger linear regression test, excluded publication bias $(P=.8)$. Meta-regression showed an insignificant variation of the effect size $(P=.5)$ during the investigated period (On-line Fig 2).

The incidence of CV was not significantly reduced among the AT-versus-control groups (79/414 versus 84/327, OR $=0.52$; $95 \% \mathrm{CI}, 0.2-1.5 ; P=.2$ ). On the other hand, the rate of good outcome during follow-up was significantly higher among the AT group compared with the control group (803/1144 versus 1175/ $1775, \mathrm{OR}=1.368 ; 95 \% \mathrm{CI}, 1.117-1.676 ; P=.002$ ). The mortality rate during follow-up was significantly reduced among the AT group $(79 / 672$ versus $97 / 571$, OR $=0.656$; 95\% CI, 0.47-0.91; $P=.01)$

Treatment-related complications were comparable among the 2 groups $(40 / 438$ versus $28 / 353, \mathrm{OR}=1.01 ; 95 \% \mathrm{CI}, 0.34-3.01$; $P=.9)$, as well as the incidence of hemorrhagic events $(32 / 438$ versus $15 / 353, \mathrm{OR}=1.7 ; 95 \% \mathrm{CI}, 0.89-3.38,0.1$ ).

\section{Subgroup Analysis}

Among the subgroup of patients treated endovascularly, there was a trend toward a lower rate of DCI after AT administration (157/ 778 versus $413 / 1410, \mathrm{OR}=0.552$; $95 \% \mathrm{CI}, 0.273-1.115 ; P=.06$ ), whereas no difference was found between patients with and without AT among the surgical group (42/195 versus 61/278, OR = 0.952; 95\% CI, 0.288-3.147; $P=.5$ ) (Table). There was a trend toward a lower incidence of DCI after long-term ( $>2$ weeks) AT (63/438 versus $96 / 353, \mathrm{OR}=0.379 ; 95 \% \mathrm{CI}, 0.12-1.2 ; P=.06)$, whereas short-term antiplatelet administration $(<2$ weeks $)$ was not associated with a significant reduction of the DCI rate (148/ 559 versus $349 / 1265, \mathrm{OR}=1.35 ; 95 \% \mathrm{CI}, 0.81-2.23 ; P=.5$ ).

Meta-regression demonstrated an association between the reduction of the rate of DCI after AT administration and the SAH grade: The higher the proportion of patients with low-grade $\mathrm{SAH}$, the lower was the incidence of DCI after AT $(P=.06)$ (Fig 2). 


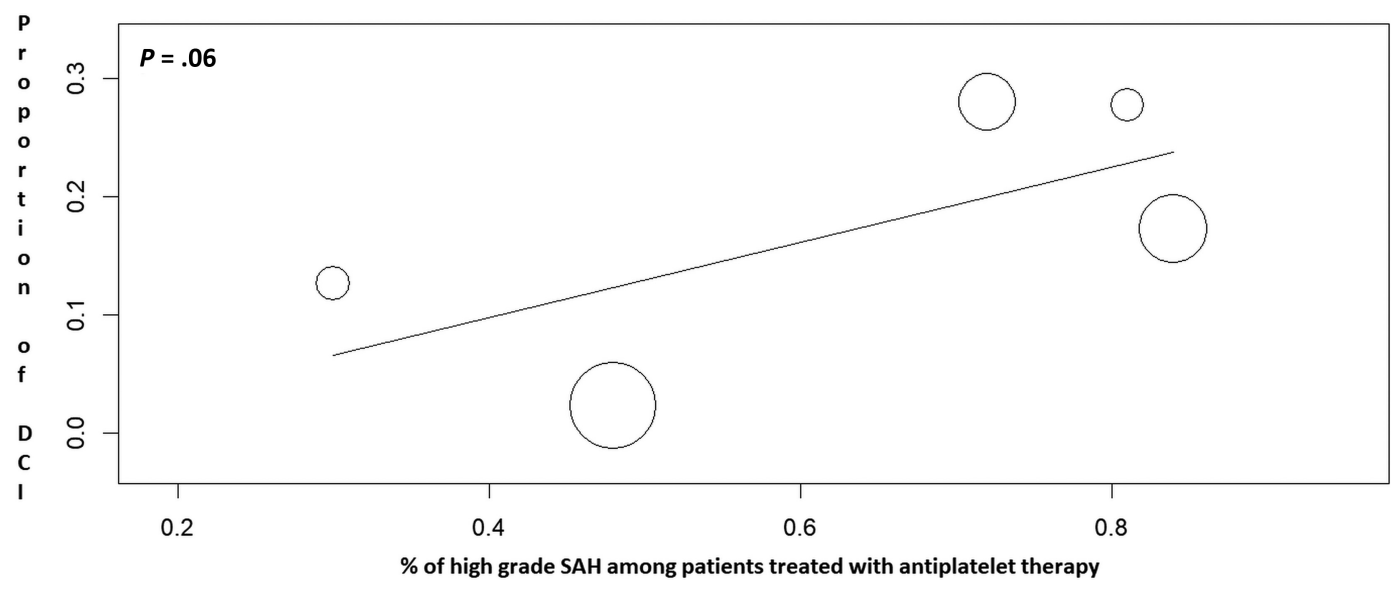

FIG 2. Meta-regression showing a trend toward a significant variation of the $\mathrm{DCl}$ rate after antiplatelet administration in relation to the proportion of patients with SAH with high-grade (Fisher 3-5) scores.

\section{Sensitivity Analysis}

Sensitivity analysis was performed with both the leave-one-out method and subgroup analysis. The leave-one-out meta-analysis showed that no individual study significantly influenced the investigated outcome (On-line Fig 3).

In addition, we divided the included studies as follows: retrospective, prospective, randomized controlled trials, and post hoc analysis from randomized controlled trials. Accordingly, we investigated the rate of DCI by performing a subgroup analysis based on the type of study (On-line Fig 4). The subgroup of retrospective studies was associated with a trend toward a lower rate of DCI among the AT group $(P=.06)$, whereas the effect of the AT was not significant among the other subgroups. The subgroup of retrospective studies included the most recent series investigating AT among the endovascularly treated patients.

\section{Study Heterogeneity}

Substantial heterogeneity $(>50 \%)$ was noted for most of the reported outcomes (rate of DCI, rate of DCI among the endovascular and surgical groups, rate of DCI after long- and short-term AT, and incidence of CV). Low heterogeneity was noted for the rates of good outcome, mortality, and complications.

\section{DISCUSSION}

Our meta-analysis of approximately 2800 patients with aneurysmal SAH highlighted several important findings about the impact of the AT on the rate of DCI and clinical outcome. Overall, this study did not show a significant beneficial effect of antiplatelet drugs on the occurrence of DCI. However, antiplatelet administration in the subgroup of patients with endovascular treatment and in the subgroup of long-term AT tended to be associated with a reduction of DCI. Despite the overall reduction of DCI not reaching a statistical significance, neurologic functional outcome in patients who received platelet inhibitors was significantly better, and mortality was significantly lower.

When we investigated the literature, data about the influence of AT on the occurrence of DCI after SAH are contradictory and quite heterogeneous. An older systematic review (2003) of clinical trials with 699 patients concluded that antiplatelet inhibitors reduced the risk of DCI, increasing the rate of good outcome in patients with SAH. ${ }^{13}$ Similarly, a more recent Cochrane review $(2007)^{14}$ indicated a trend toward better outcome and a lower incidence of DCI among patients treated with antiplatelet agents after SAH. However, the results were not statistically significant, and no definite conclusions were reached.

Studies included in these reviews had the following characteristics: 1) All series reported surgically treated patients, with only 1 study reporting both endovascular and surgical treatments; 2) different types of antiplatelet agents were administrated (ASA, OKY, dipyridamole, ticlopidine); 3) some older series (before 1990) did not specifically investigate the incidence of DCI, reporting a general rate of neurologic deficit after treatment; and 4) other drugs, such as tranexamic acid, were used in some series in combination with ASA. Our study, the largest to date, presents more specific selection criteria: 1) Ninety-five percent of patients were treated with ASA, with only 1 very recent study reporting treatment with ASA + clopidogrel ${ }^{8}$; 2) DCI was the primary investigated outcome with a clear radiologic and clinical definition; and 3) $75 \%$ of patients were treated endovascularly, while $25 \%$ underwent surgical clipping (this is a more representative proportion of the modern treatment management of ruptured intracranial aneurysms); and finally 4) the effect of the AT was investigated in the endovascular and surgical groups, as well as in relation to the duration of the AT.

We found that the reduction of DCI $(\mathrm{OR}=0.78, P=.33)$ and vasospasm $(\mathrm{OR}=0.52, P=.2)$ after antiplatelet administration was not statistically significant. However, patients receiving antiplatelet agents had a better clinical outcome $(\mathrm{OR}=1.36, P=$ $.002)$ and lower mortality rate $(\mathrm{OR}=0.65, P=.01)$. Reasons behind this benefit are difficult to explain, ${ }^{5}$ but antiplatelet drugs can be associated with a decreased occurrence of microemboli. Microemboli have been detected in both clinical series ${ }^{6}$ and autoptic studies of patients with $\mathrm{SAH},{ }^{7}$ representing a parallel pathogenic mechanism impacting the cerebral microcirculation and the neurologic outcome..$^{5,7}$

Similarly, in the Magnesium and Acetylsalicylic acid in Subarachnoid Hemorrhage (MASH) trial, ${ }^{5}$ there was not a significant beneficial effect of ASA on the incidence of DCI, despite ASA treatment resulting in a better functional outcome at follow-up. 
The authors mentioned different factors that are likely associated with the lack of effect of ASA: 1) ASA alone, and the dose of aspirin (100 mg), might not be enough; 2) nearly $70 \%$ of patients were assigned to surgery, and it has been demonstrated that clipping might increase the risk of DCI compared with coiling ${ }^{15}$; and 3) ASA might not be the right antiplatelet drug. Dorhout Mees et $\mathrm{al}^{13}$ in a meta-analysis of randomized trials, found a beneficial effect of AT mainly based on trials with antiplatelet agents different from aspirin. In addition, in the MASH trial, aspirin was given for 2 weeks after SAH. Our study found a trend toward lower DCI $(\mathrm{OR}=0.37, P=.06)$ among patients treated with AT for longer than 2 weeks compared with those receiving antiplatelet drugs for a shorter period ( $\leq 2$ weeks). In general, arterial narrowing starts 3-5 days after SAH, with the highest incidence of DCI and vasospasm between days 5 and 14 and gradually resolving between 2 and 4 weeks. ${ }^{16,17}$ Accordingly, it is likely that 2 weeks of AT might not give protection for the overall period of risk for CV and DCI (after 14 days).

\section{Endovascular-versus-Surgical Groups}

In a post hoc analysis of the MASH trial, van den Bergh et al ${ }^{11}$ investigated the influence of aspirin on the subgroup of patients with SAH treated with coiling. The analysis found a relative risk of 1.3 (DCI clip/DCI coil ratio) in favor of endovascular treatment: The result implies that patients with endovascular coiling had a higher reduction of DCI after aspirin, compared with patients with neurosurgical clipping. Similarly, a recent series of patients treated endovascularly reported a significant positive impact of antiplatelet administration on the DCI risk. Darkwah Oppong et $\mathrm{al}^{2}$ reported a series of 329 patients with ruptured aneurysms treated with endovascular techniques and receiving antiplatelet medication: ASA was independently associated with a reduced DCI risk (adjusted OR $=0.41, P<.001$ ). In a very recent series of 161 patients with aneurysmal SAH treated by endovascular means, 85 received dual-antiplatelet therapy (ASA + clopidogrel) because of the use of additional stents, whereas 76 were allocated to the control group. The risk of clinical vasospasm $(\mathrm{OR}=0.2$, $P=.003)$ and $\mathrm{DCI}(\mathrm{OR}=0.056, P=.001)$ was significantly lower in patients receiving dual-antiplatelet therapy. ${ }^{8}$ Accordingly, our meta-analysis found a trend toward a reduced incidence of DCI after antiplatelet treatment among the endovascular group $(\mathrm{OR}=$ $0.55, P=.06$ ), but the reduction was not significant among the surgical group ( $\mathrm{OR}=0.95, P=.5$ ). A large Cochrane metaanalysis ${ }^{18}$ comparing endovascular-versus-surgical treatment reported that DCI after SAH was observed in 292/1225 (24\%) participants allocated to the endovascular group and in $349 / 1225$ (28\%) participants allocated to the surgical treatment group, with a relative and absolute risk reduction of coiling versus clipping of $16 \%$ (risk reduction, 0.84 ) and $4 \%$, respectively. In the International Subarachnoid Aneurysm Trial (ISAT) ${ }^{15}$ clipping increased the risk of DCI compared with coiling in the 2143 patients $(\mathrm{OR}=$ 1.124). Expert opinions reported that manipulation of the brain and vessel wall during the operation increases the risk of vasospasm and DCI, though there is no reliable evidence supporting this theory. ${ }^{19}$ In conclusion, part of the reduction of DCI might be explained by a combination of the treatment (endovascular) and the use of antiplatelet drugs.

\section{Relationship between the Grade of SAH and DCI Reduction after $A T$}

The overall reduction of DCI after antiplatelet administration tended ( $P=.06$ ) to be directly related to the proportion of patients with SAH with a low-grade (Fisher 1-2) score: In other words, the higher the SAH grade, the lower was the effect of antiplatelet agents on the DCI reduction (Fig 2). In general, the SAH grade is an independent risk factor for vasospasm and $\mathrm{DCI},{ }^{20}$ and it is likely that AT had a lower effect among patients with larger amounts of cisternal blood. Finally, AT during endovascular treatment of acutely ruptured intracranial aneurysms might increase the risk of ventriculostomy-related hemorrhages. A recent meta-analysis ${ }^{21}$ reported that ventriculostomy-related hemorrhage rates were $20 \%$ and $9 \%$ among patients with SAH treated with and without AT, respectively, though major hemorrhages were low in both groups ( $4.4 \%$ and $1 \%$, respectively).

\section{Strength and Limitations}

Our study has limitations. First, the heterogeneity among studies was high for most of the reported outcomes; this is likely related to the different treatment techniques and different doses of aspirin. Accordingly, our results should be interpreted with caution. The influence of the dose of AT was not investigated because the available data did not allow performing subgroup analysis. The $P$ values of the subgroup analysis (endovascular-versus-surgical groups, long-term-versus-short-term AT) were around .05. Consequently, the results presented a certain degree of evidence, ${ }^{22}$ but they did not reach statistical significance. However, publication bias was reasonably excluded, and our study is the largest to date analyzing the use of antiplatelet drugs among patients with SAH.

\section{CONCLUSIONS}

In our study, the overall incidence of DCI among patients with $\mathrm{SAH}$ seems not to be significantly reduced after antiplatelet drug administration. However, DCI tended to be lower, both among subjects with long-term ( $>2$ weeks) AT and patients with endovascular treatment and antiplatelet administration. In addition, patients with AT and lower amounts of cisternal blood tend to have a lower incidence of DCI. Finally, poor outcome and mortality rates were significantly reduced among the AT group. Future randomized trials are needed to assess the benefits of AT among individuals with $\mathrm{SAH}$ allocated to the endovascular treatment.

Disclosures: Paolo Perrini-UNRELATED: Employment: University of Pisa, Comments: consultant neurosurgeon at University of Pisa. Vincent Costalat-UNRELATED: Consultancy: Balt, MicroVention, Medtronic; Grants/Grants Pending: Medtronic, Stryker*; Payment for Development of Educational Presentations: Balt, Medtronic, Stryker, MicroVention. Pierre-Henri Lefevre-UNRELATED: Payment for Development of Educational Presentations: Medtronic. Alain Bonafe-UNRELATED: Consultancy: Medtronic, Stryker, MicroVention. * Money paid to the institution.

\section{REFERENCES}

1. Budohoski KP, Guilfoyle M, Helmy A, et al. The pathophysiology and treatment of delayed cerebral ischaemia following subarachnoid haemorrhage. J Neurol Neurosurg Psychiatry 2014;85:1343-53 CrossRef Medline

2. Darkwah Oppong M, Gembruch O, Pierscianek D, et al. Post-treatment antiplatelet therapy reduces risk for delayed cerebral isch-

AJNR Am J Neuroradiol 40:1201-06 Jul 2019 www.ajnr.org

1205 
emia due to aneurysmal subarachnoid hemorrhage. Neurosurgery 2018 Dec 13. [Epub ahead of print] CrossRef Medline

3. Hop JW, Rinkel GJ, Algra A, et al. Randomized pilot trial of postoperative aspirin in subarachnoid hemorrhage. Neurology 2000;54: 872-78 CrossRef Medline

4. Juvela S. Aspirin and delayed cerebral ischemia after aneurysmal subarachnoid hemorrhage. J Neurosurg 1995;82:945-52 CrossRef Medline

5. van den Bergh WM, Algra A, Dorhout Mees SM, et al; MASH Study Group. Randomized controlled trial of acetylsalicylic acid in aneurysmal subarachnoid hemorrhage: the MASH study. Stroke 2006;37: 2326-30 CrossRef Medline

6. Romano JG, Forteza AM, Concha M, et al. Detection of microemboli by transcranial Doppler ultrasonography in aneurysmal subarachnoid hemorrhage. Neurosurgery 2002;50:1026-30; discussion 1030-31 Medline

7. Stein SC, Browne KD, Chen XH, et al. Thromboembolism and delayed cerebral ischemia after subarachnoid hemorrhage: an autopsy study. Neurosurgery 2006;59:781-87; discussion 787-88 CrossRef Medline

8. Nagahama $Y$, Allan L, Nakagawa D, et al. Dual antiplatelet therapy in aneurysmal subarachnoid hemorrhage: association with reduced risk of clinical vasospasm and delayed cerebral ischemia. J Neurosurg 2018;129:702-10 CrossRef Medline

9. Moher D, Liberati A, Tetzlaff J, et al. Preferred reporting items for systematic reviews and meta-analyses: the PRISMA statement. Int J Surg 2010;8:336-41 CrossRef Medline

10. Wells G, Shea B, O'Connell D, et al. The Newcastle-Ottawa Scale (NOS) for assessing the quality of nonradomized studies in metaanalyses. Ottawa: Ottawa Hospital Research Institute, 2011. http:// www.ohri.ca/programs/clinical_epidemiology/oxford.asp. Accessed February 22, 2019

11. van den Bergh WM, Algra A, Rinkel GJ, et al; MASH Study Group. Magnesium and aspirin treatment in patients with subarachnoid haemorrhage: comparison of effects after endovascular and neurosurgical aneurysm occlusion. J Neurol 2009;256:213-16 CrossRef Medline

12. van den Bergh WM, Kerr RS, Algra A, et al; International Subarach- noid Aneurysm Trial (ISAT) Collaborative Group. Effect of antiplatelet therapy for endovascular coiling in aneurysmal subarachnoid hemorrhage. Stroke 2009;40:1969-72 CrossRef Medline

13. Dorhout Mees SM, Rinkel GJ, Hop JW, et al. Antiplatelet therapy in aneurysmal subarachnoid hemorrhage: a systematic review. Stroke 2003;34:2285-89 CrossRef Medline

14. Dorhout Mees SM, van den Bergh WM, Algra A, et al. Antiplatelet therapy for aneurysmal subarachnoid haemorrhage. Cochrane $D a$ tabase Syst Rev 2007;CD006184 Medline

15. Dorhout Mees SM, Kerr RS, Rinkel GJ, et al. Occurrence and impact of delayed cerebral ischemia after coiling and after clipping in the International Subarachnoid Aneurysm Trial (ISAT). J Neurol 2012; 259:679-83 CrossRef Medline

16. Vergouwen MD, Vermeulen M, van Gijn J, et al. Definition of delayed cerebral ischemia after aneurysmal subarachnoid hemorrhage as an outcome event in clinical trials and observational studies: proposal of a multidisciplinary research group. Stroke 2010; 41:2391-95 CrossRef Medline

17. Weir B, Grace M, Hansen J, et al. Time course of vasospasm in man. J Neurosurg 1978;48:173-78 CrossRef Medline

18. Lindgren A, Vergouwen MD, van der Schaaf I, et al. Endovascular coiling versus neurosurgical clipping for people with aneurysmal subarachnoid haemorrhage. Cochrane Database Syst Rev 2018;8: CD003085 CrossRef Medline

19. Brilstra EH, Rinkel GJ, Algra A, et al. Rebleeding, secondary ischemia, and timing of operation in patients with subarachnoid hemorrhage. Neurology 2000;55:1656-60 CrossRef Medline

20. Lee H, Perry JJ, English SW, et al. Clinical prediction of delayed cerebral ischemia in aneurysmal subarachnoid hemorrhage. J Neurosurg 2018 Jun 1:1-8. [Epub ahead of print] CrossRef Medline

21. Cagnazzo F, Di Carlo DT, Petrella G, et al. Ventriculostomy-related hemorrhage in patients on antiplatelet therapy for endovascular treatment of acutely ruptured intracranial aneurysms: a meta-analysis. Neurosurg Rev 2018 Jul 2. [Epub ahead of print] CrossRef Medline

22. Wood J, Freemantle N, King M, et al. Trap of trends to statistical significance: likelihood of near significant $P$ value becoming more significant with extra data. BMJ 2014;348:g2215 CrossRef Medline 